\title{
Filtering Data Streams for Entity-Based Continuous Queries
}

\author{
Reynold Cheng, Member, IEEE, Ben C.M. Kao, Alan Kwan, \\ Sunil Prabhakar, and Yi-Cheng Tu, Member, IEEE
}

\begin{abstract}
The idea of allowing query users to relax their correctness requirements in order to improve performance of a data stream management system (e.g., location-based services and sensor networks) has been recently studied. By exploiting the maximum error (or tolerance) allowed in query answers, algorithms for reducing the use of system resources have been developed. In most of these works, however, query tolerance is expressed as a numerical value, which may be difficult to specify. We observe that in many situations, users may not be concerned with the actual value of an answer, but rather which object satisfies a query (e.g., "who is my nearest neighbor?"). In particular, an entity-based query returns only the names of objects that satisfy the query. For these queries, it is possible to specify a tolerance that is "nonvalue-based." In this paper, we study fraction-based tolerance, a type of nonvalue-based tolerance, where a user specifies the maximum fractions of a query answer that can be false positives and false negatives. We develop fraction-based tolerance for two major classes of entity-based queries: 1) nonrank-based query (e.g., range queries) and 2) rank-based query (e.g., $k$-nearest-neighbor queries). These definitions provide users with an alternative to specify the maximum tolerance allowed in their answers. We further investigate how these definitions can be exploited in a distributed stream environment. We design adaptive filter algorithms that allow updates be dropped conditionally at the data stream sources without affecting the overall query correctness. Extensive experimental results show that our protocols reduce the use of network and energy resources significantly.
\end{abstract}

Index Terms-Data streams, continuous queries, adaptive filters, fraction-based tolerance.

\section{INTRODUCTION}

$\mathrm{D}^{2}$ UE to the rapid development of low-cost sensors and networking technologies, stream applications have attracted tremendous research interests lately. In particular, long-standing continuous queries are common in a stream environment for monitoring various network activities. Some examples include intrusion detection over securitysensitive regions; identification of Denial-of-Service (DOS) attacks on the Internet [4]; road traffic monitoring; natural habitat monitoring; network fault detection; e-mail spams detection; and Web statistics collection.

In such applications, stream sources are installed to collect and report the states of various entities. A large number of sources (e.g., GPS-enabled devices, Internet hosts, and wireless sensors) report their updated values (e.g., locations, TCP packets, and temperature values) continuously to the processing server. These systems often have limited bandwidth or energy resources. For example, in sensor networks, sensing devices usually have scarce battery power and communicate in a low-bandwidth environment.

- R. Cheng, B.C.M. Kao, and A. Kwan are with the Department of Computer Science, The University of Hong Kong, Pokfulam, Hong Kong.

E-mail: \{ckcheng, kao, klkwan\}@cs.hku.hk.

- S. Prabhakar is with the Department of Computer Science, Purdue University, 250 N. University Street, West Lafayette, IN 47907.

E-mail: sunil@cs.purdue.edu.

- Y.-C. Tu is with the Department of Computer Science and Engineering, University of South Florida, 4202 E. Fowler Ave., ENB118, Tampa, FL 33620.E-mail:ytu@cse.usf.edu.

Manuscript received; 13 Oct. 2007; revised 13 June 2008; accepted 5 Feb. 2009; published online 25 Feb. 2009.

Recommended for acceptance by S. Chakravarthy.

For information on obtaining reprints of this article, please send e-mail to tkde@computer.org, and reference IEEECS Log Number TKDE-2007-10-0504. Digital Object Identifier no. 10.1109/TKDE.2009.63.
Moreover, since the number of stream sources could be large, a stream server could be crippled by the large volume of data. This slows its response to standing queries that require real-time processing [3]. It is thus important to lower the message volume so that transmission cost as well as server's load can be reduced.

One direct way of lowering transmission cost is to drop some of the data items generated from the stream sources. The drawback is that the server may have to process queries based on inaccurate data. However, if we can carefully select the items to be dropped, the accuracy of a query answer may only be affected slightly. Consider a transportation monitoring system, where vehicles within a geographical region (e.g., the city center) can be tracked [35]. For vehicles that are stationary, or are located far away from the region, it may not be necessary for these vehicles to always report their positions to the system (in order to have high query accuracy). More generally, for many standing queries, a user may accept an answer with the maximum error allowed (or tolerance) in exchange for lower resource consumption and better timeliness in query processing. Other examples for which controlled query errors are acceptable include wide-area resource accounting and load balancing in replicated servers. Intelligent protocols have been proposed [28], [19] to wisely control when stream sources should report updates. The goal of the protocols is to reduce communication overhead, while at the same, time userspecified tolerances are met. These protocols make use of filter bounds-a system-specified range of values. A stream source only reports an update if its value crosses the bound.

To illustrate the above concepts, let us consider a continuous range query, a service commonly found in location-based and sensor applications. In this query, a 


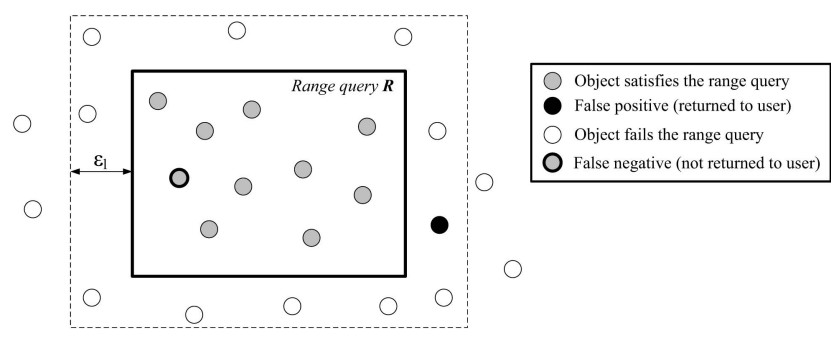

Fig. 1. Illustrating query tolerances.

user is interested in stream values that fall within a region called query range. This query can be used in an intelligent transportation system, which monitors the movement of GPS-enabled vehicles inside a geographical region for an extensive amount of time. Another example is wildlife tracking, which allows real-time analysis of the movement of animals that gather around a landscape structure, e.g., a water hole [20]. The animals, attached with location sensors, can be monitored for a month's time by a continuous range query with the surrounding area of the water hole as a query range. Fig. 1 illustrates a continuous range query over locations of moving objects. A rectangle $R$ (in solid lines) is specified by the user. The query returns the identities of objects whose locations are inside $R$ (these objects are colored in gray).

How would a user express a tolerance for this query? One possibility is to let the user choose a numerical tolerance, say $\epsilon$, and the system guarantees that the ID of any object returned to the user must be located inside the dashed-line rectangle (but not necessarily inside $R$ ). This kind of tolerance, expressed as a numerical value, is often assumed by filter-bound-based approximation techniques. In Fig. 1, the filter bound is exactly the dashed-line rectangle and installed in each moving object. This means that even if an object is outside $R$, it needs not report its location to the system as long as it does not cross the filter bound.

While numerical tolerance is useful, choosing an appropriate value of it may not be straightforward. In particular, specifying a numerical tolerance requires some knowledge about the relative distances or spread of the objects. For instance, should $\epsilon$ be 1 meter or 100 meters? In a sensor network, various kinds of data such as humidity, temperature, and UV-index are collected [12]. If only a numerical tolerance is allowed, the user may need to know a reasonable range of error for each data type. Also, if a data stream contains multidimensional data (e.g., location, speed) or multimedia data (e.g., images), a numerical, or value-based error, could be difficult to specify. A bad choice of the numerical tolerance may significantly weaken the value of a query. From Fig. 1, we can see that a large number of objects whose locations are outside $R$ (i.e., those colored in black and white) are also included in the answer. To solve this problem, a user has to be careful not to set $\epsilon$ too large. However, if $\epsilon$ is too small, it may not be very useful for improving the system performance. Thus, finding a reasonable value of $\epsilon$ can be difficult.

Alternatively, query tolerance can be expressed in terms of a fraction rather than an absolute value. To illustrate, Fig. 1 shows an object, colored in black, that is included in the answer although the object is not inside $R$. This object is called a false positive [27]. A fraction-based tolerance can then be defined as the maximum fraction of query answers that can be false positives. For example, if the fraction-based tolerance is 0.1 , then the black-colored object can still be included in the answer, which contains nine other objects. Another possibility to express the fraction-based tolerance is through the use of false negatives [27], which specifies the maximum fraction of objects that belong to the query result but are not included in the answer returned to the user. An example of a false negative is illustrated as a circle with a thick boundary in Fig. 1; although it is inside $R$, it is not included in the user's result. Notice that the concepts of false positives/negatives are based on precision and recall in the IR literature [17].

The main goal of this paper is to study how filter bounds can be deployed in data stream sources in order to exploit fraction-based tolerance for different continuous query types. Let us use the range query example in Fig. 1 again to illustrate our solutions. Based on the maximum fraction of false positives allowed, we first compute the number $m$ of stream sources that can be false positives. Then, $m$ stream sources that are currently satisfying the query are requested to stop sending their data to the system. For the remaining streams that satisfy the query, they are assigned with the filter bound with the range $R$. Notice that regardless of whether the values of the $m$ "shutdown" stream sources satisfy the range query, the query answer is still acceptable within the tolerance. Hence, as long as no updates are received by the system, the query answer remains correct with respect to the tolerance. Similarly, a maximum number of false negative stream sources are stopped from reporting updates. Since only the stream sources with the filter bound $R$ send their updates when their values cross the bound, our protocol saves network and energy costs of data transmission.

Besides range query, we also study filter bound protocols for a rank-based query, another important query type in streaming applications. Contrast to a range query, a rankedbased query returns IDs of objects based on their relative rankings. For example, in a transportation system, a longstanding $k$-nearest-neighbor ( $k$-NN) query can be issued, which continuously returns the IDs of $k$ vehicles closest to a given query point [18] for a long period of time. In a habitat monitoring system, scientists may be interested in tracking the $k$ areas that yield the highest temperature, in which case a top- $k$ query can be used [12]. As another example, in network traffic analysis, it is important to identify heavy hitters [5], [15]. A heavy hitter is an IP source that delivers a large number of packets to the monitored network, which is also likely involved in DOS attacks. If the user is interested in monitoring the $k$ sources that yield the largest traffic volume, then a continuous top- $k$ query can be used. Notice that both the range query (a nonrank-based query) and the $k$-NN/top- $k$ query (a rank-based query) return sets of object IDs, rather than numerical values as answers. These entitybased queries are good candidates for using fraction-based tolerance, which does not use numerical values.

A few technical challenges need to be addressed for filter bound protocols that exploit fraction-based tolerance. First, as we will show, when value updates are received from stream sources, the tolerance requirement can be violated 
(e.g., the fraction of false positives is larger than the allowed threshold). We tackle this problem by carefully adjusting the filter bounds of the stream sources so that query correctness can be restored. Second, this correctness maintenance process, which involves several message exchanges between the server and the stream sources, can be expensive. Moreover, some stream sources that are considered as false positives/negatives and are not involved in data transmission may have to be "waken up" to send data in order to maintain query correctness. We propose two new techniques, namely, incremental deployment and immediate compensation, in order to address these problems. The incremental deployment technique reduces the chance of expensive error fixing by only allowing a fraction of false positive/negative stream sources to be "shut down." The immediate compensation method is designed to force an active data stream source to stop transmitting data, in order to compensate the "waking-up" of another stream source source.

While most previous works in filter bound algorithms assume value-based queries (e.g., SUM and AVG), we study the entity-based queries. We investigate the computation and communication costs of our protocols. We also perform simulations to examine the effectiveness of our protocols. Although we assume one-dimensional data here, our techniques can be generalized to higher dimension cases. To summarize, our contributions are:

- Define fraction-based tolerance for rank-based and nonrank-based queries,

- Present protocols that exploit fraction-based tolerances,

- Derive the computation, communication, and energy costs of the protocols, and

- Perform extensive experiments on the protocols, using both real and synthetic data.

The rest of this paper is organized as follows: We discuss related work in Section 2. Section 3 defines the semantics of fraction-based tolerance constraints. Section 4 presents protocols for maintaining filter constraints for fraction-based tolerance of nonrank-based queries. Then, Section 5 explains how the nonrank-based query protocol can be extended to support rank-based query. In Section 6, we analyze the resource consumption for our protocols and present our experimental results. We conclude the paper in Section 7.

\section{Related Works}

Tolerance classification. Due to the high volume and continuous nature of data streams, an important goal of a stream management system is to conserve system resources such as battery [13], memory [3], computation [22], [29], [16], and communication costs [14], [28], [19]. Most of these works reduce resource consumption by relaxing correctness requirements. Typically, a user specifies a maximum tolerance, and the tolerance is exploited by various techniques such as approximate data structures, load shedding, filters, etc. The tolerance is often assumed to be in the form of a numerical value. Also, they are mostly applicable to value-based queries only. Our work investigates the possibility of exploiting fraction-based tolerance, a type of nonvaluebased tolerance, for continuous entity-based queries.

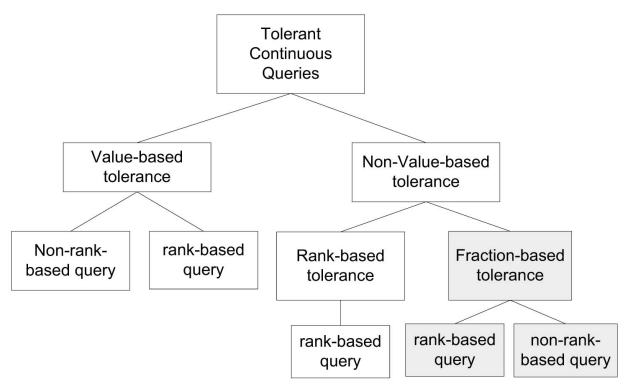

Fig. 2. Classification of query tolerances.

Fig. 2 illustrates a classification of tolerances. The rankbased tolerance, which describes the error in terms of the distance from the desired order to the actual answer, is studied in [7]. For example, given that the query returns the object with the largest value, the user can specify that he can accept an object which ranks the second or the third. We extend the study of fraction-based tolerance maintenance proposed in [7]. This tolerance, as shaded in Fig. 2, has a broader application than rank-based tolerance, since it can be applied to both rank- and nonrank-based queries.

Adaptive filters. The idea of using adaptive filters in which filter bounds are installed to reduce communication costs was first proposed in [28]. However, that paper only considers value-based tolerance over aggregate queries such as average value and minimum value. In [4], a similar idea is applied to answer top- $k$ queries for distributed stream sources, but again, the tolerance is value-based. In [19], Kalman Filters are used to exploit value-based tolerance. The Kalman Filter is installed at every stream source, and with its prediction techniques, it is shown to be more effective than previous methods in conserving communication costs. The extension of adaptive filters in a sensor network is studied in [11]. Our work differs from theirs in that we use adaptive filters to exploit nonvaluebased tolerance. In addition, we study continuous $k-\mathrm{NN}$ queries that are used in applications such as computeraided manufacturing and traffic monitoring [22]. Notice that $k$-NN queries are more general than top- $k$ queries studied in [19]. Adaptive filters for $k$-NN queries are also studied in [26], but the use of nonvalue-based tolerance is again not considered.

Nonvalue-based tolerance. The classification of queries into value-based and entity-based has been proposed in [6]. To our best knowledge, the use of nonvalue-based tolerance for entity-based queries has not been well studied. In [34], approximate answers for set-valued queries are proposed, where a query answer contains a set of objects. An exact answer $E$ is approximated by two sets: a certain set $C$ which is a subset of $E$ and a possible set $P$ such that $C \cup P$ is a superset of $E$. This notion can be used to generate approximate query results if a portion of the relational database is unavailable, or if there is not enough time to produce an exact answer. A rank precision model is proposed in [21]: an answer $a$ is called $\alpha$-precise if the true rank of $a$ lies in the interval $[r-\alpha, r+\alpha]$, where $r$ is the rank of $a$ informed to the user. In [10], precision and recall are used as quality metric for approximating $k$-NN queries. The issues of defining and exploiting fraction-based tolerance in stream systems have only been addressed in [7]. However, that work uses a preliminary protocol to 
handle the tolerance. Here, we propose a more efficient protocol, which performs better than that in [7]. We also examine energy consumption issues, which have not been studied before.

Other query evaluation techniques. The idea of viewing a $k$-NN query as a range query was proposed in [18]. They use a bound which encloses at least $k$ objects so that continuous $k$-NN queries can be answered efficiently. For our filter bound protocol for continuous $k$-NN query, we also convert the query to a range query.

In [25], a cost-based algorithm for MAX/MIN query was proposed for sensor environments. The algorithm provides a provably smallest cost in probing the data from the stream sources. Their method is only used for "snapshot queries" (i.e., queries that are executed once only), whereas our approach (which uses adaptive filters) is designed for continuous queries. We also study the effectiveness in energy consumption, which is not studied in that paper.

Another cost-based approach for sensor network monitoring was recently studied in [30]. Their energy consumption model used for optimization is described in terms of message cost. Specifically, for every $x$-byte message sent, $\sigma+\delta x$ units of energy will be consumed, where $\delta x$ is the energy spent for sending $x$ bytes, and $\sigma$ is a per-message overhead. In our paper, we further exploit the fact that sensors can be in "sleep mode," which consumes a minimal amount of energy. By using fraction-based tolerance, we show that it is possible to save energy by allowing a controlled portion of stream sources to be in the sleep mode.

Load shedding. In this method, the database server drops data tuples that arrived at the server site, in order to lower the resource demand. Query processing also has to be accomplished under some Quality-of-Service (QoS) requirements [31], [32], [8], [1]. Although filtering techniques also attempt to reduce resource utilization by dropping tuples, they are generally different from load shedding. For filtering, the dropping of tuples occurs in stream sources rather than in the server. Hence, filtering can save more communication costs.

\section{Problem Definition}

We assume a distributed stream management model similar to those described in [4], [28], [19]. The system consists of a set $S=\left\{S_{1}, \ldots, S_{i}, \ldots, S_{n}\right\}$ of $n$ data stream sources with stream source $S_{i}$ reporting a value $v_{i} \in \Re$. We assume that stream values are updated at discrete time instants. Each stream source may be associated with an adaptive filter that specifies a constraint. With the filter mechanism, not all updates are reported to the server. A filter constraint is a closed interval $\left[l_{i}, u_{i}\right]$, where $l_{i}, u_{i} \in \Re$. Let $v_{i}^{\prime}$ be the last reported value from stream source $S_{i}$. When the stream's value $\left(v_{i}\right)$ changes, the filter constraint is violated if either 1) $v_{i}^{\prime} \in\left[l_{i}, u_{i}\right] \wedge v_{i} \notin\left[l_{i}, u_{i}\right]$ or 2$) v_{i}^{\prime} \notin\left[l_{i}, u_{i}\right] \wedge v_{i} \in\left[l_{i}, u_{i}\right]$. Only when the constraint is violated will the updated value be sent to the server. If no filter is installed at a stream source, all updates from the stream source are reported.

Fig. 3 shows a general architecture of such systems. Each stream source is equipped with a filter that is adaptive whose parameters can be changed at any time by the processor. A user submits her queries and tolerance requirements to the central processor. The constraint assignment unit then determines the relevant filter constraints to be installed in

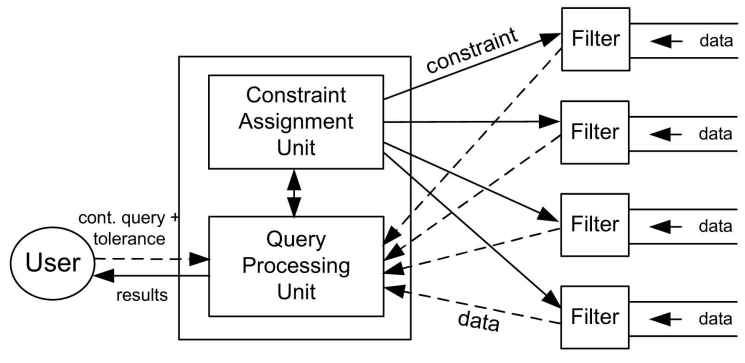

Fig. 3. Data stream management system model.

each stream source. The query processing unit processes user queries and updates their results if necessary. It also receives updates from the stream sources. It communicates with the constraint assignment unit, which decides if constraints need to be revised for relevant filters.

\subsection{Query Model}

We are interested in entity-based queries-those that return identifiers of objects as answers [6]. We classify entity-based queries into rank-based queries and range queries.

1. Rank-based query. Given a number $k \in \aleph(k$ is called the rank requirement), a rank-based query returns IDs of objects that rank $k$ th or above. Here, we use $k$-NN queries to illustrate filter protocols, since such queries are common in systems like computer-aided manufacturing (CAM) in a productline monitoring system, mobile environments, and network traffic monitoring [18], [22], [10]. A CAM, for example, uses $k$-NN queries to discover similar patterns over multidimensional data obtained from sensors installed in production lines. A $k$-NN query can also answer $k$-min and $k$-max queries. Notice that a $k$-min ( $k$-max) query is just a $k$-NN query by setting the query point $q$ to $-\infty$ (respectively, $+\infty$ ).

2. A range query is specified by an interval $[l, u]$. Streams whose values fall within $[l, u]$ are returned to the user. A range query is nonrank-based since the decision of whether a stream contributes an answer is independent of others.

Here, we use $Q$ to denote an entity-based standing query and $A(t)$ to denote the answer set returned at time $t$. We use $|A(t)|$ to denote the cardinality of $A(t)$.

A standing query $Q$ is associated with a tolerance constraint. We focus on the fraction-based tolerance, a kind of nonvalue-based tolerance. The remaining of this section presents and explains the definition of this tolerance.

\subsection{Fraction-Based Tolerance}

As explained, fraction-based tolerance adopts the concept of false positives and negatives. This tolerance applies to all entity-based queries, i.e., both rank- and nonrank-based queries. An example of fraction-based tolerance for nonrank-based queries is the reporting of alert messages about network sources, which yield a traffic volume within an abnormal range. For security purposes, it may be acceptable that these messages are sent to the network administrator even if the message is a false alarm. The network source wrongly reported can then be regarded as a false positive. As for rank-based queries, consider a CAM system [22], where 

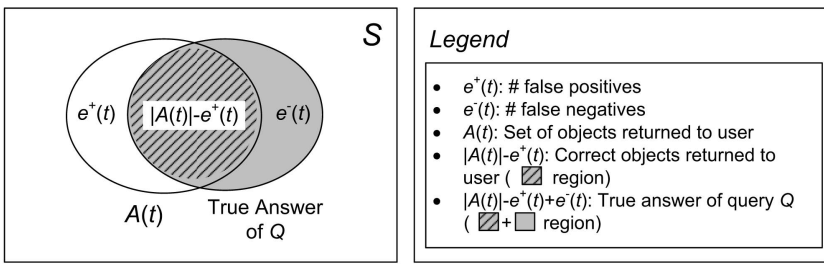

Fig. 4. Illustrating $A(t), e^{+}(t)$, and $e^{-}(t)$.

feedback information is returned from production lines to adjust the parameters. In these systems, sensors are installed to monitor the parts and discover the patterns of similar features. Users issue the $k$-NN queries to mine multimedia data streams (e.g., images) for unknown patterns, where the features are multidimensional such as position, shape, size, surface characterization, material properties, etc. Those features are difficult to specify based on a numerical tolerance (because the user may not have a sense of how much the error value should be, as discussed in Section 1). A fraction-based tolerance, on the other hand, is more intuitive in quantifying the quality of results [10]. Based on the fraction-based tolerance, the system will trigger an alert if certain percentage of discrepancies in the result is reached.

Definition 1 (False positive and false negative). Given query $Q$ and answer set $A(t)$ returned to the user, let $e^{+}(t)$ denote the number of streams in $A(t)$ that fail $Q$, and $e^{-}(t)$ be the number of streams that satisfy $Q$ but are not in $A(t)$. The fraction of false positives and the fraction of false negatives of $Q$ at time $t$, denoted by $f^{+}(t)$ and $f^{-}(t)$, respectively, are:

$$
\begin{gathered}
f^{+}(t)=\frac{e^{+}(t)}{|A(t)|}, \\
f^{-}(t)=\frac{e^{-}(t)}{|A(t)|-e^{+}(t)+e^{-}(t)} .
\end{gathered}
$$

Equation (1) describes the portion of objects returned to the user that are not correct (false positives). Equation (2) is essentially the fraction of objects in the true query answer that are not returned to the user (i.e., false negatives). The denominator of (2) (i.e., $|A(t)|-e^{+}(t)+e^{-}(t)$ ) is the size of the true answer set, where the false positives that do not satisfy $Q$ are excluded and false negatives that satisfy $Q$ but not in $A(t)$ are included. Fig. 4 illustrates the relationship among these quantities. Notice that the notions of false positives/negatives are based on the concept of precision and recall [17]. In particular, $f^{+}(t)=1-$ precision, and our goal can be viewed as achieving the minimum precision required for query answers. Similarly, $f^{-}(t)=1-$ recall.

Definition 2 (Fraction-based Tolerance). Given query $Q$, answer set $A(t)$, maximum false positive tolerance $\epsilon^{+}$, and maximum false negative tolerance $\epsilon^{-}$, the answer set $A(t)$ is correct w.r.t. $\epsilon^{+}$and $\epsilon^{-}$iff $f^{+}(t) \leq \epsilon^{+}$and $f^{-}(t) \leq \epsilon^{-}$.

The parameters $\epsilon^{+}$and $\epsilon^{-}$are user-specified. The system has to guarantee that the fraction-based tolerances are met. We assume that $\epsilon^{+}$and $\epsilon^{-}$are both smaller than 0.5 , because in most scenarios, users are not interested in results with more wrong answers than correct ones. This assumption is also required for guaranteeing the correctness of our protocols.
Let $e^{\max +}(t)$ be the maximum number of answers that can be incorrect in $A(t)$ and $e^{\max -}(t)$ be the maximum number of stream sources that satisfy the query but are excluded from $A(t)$. From (1) and (2), we have

$$
f^{+}(t) \leq \frac{e^{\max +}(t)}{|A(t)|}=\epsilon^{+},
$$

$$
f^{-}(t) \leq \frac{e^{\max -}(t)}{|A(t)|-e^{\max +}(t)}=\epsilon^{-} .
$$

More about fraction-based tolerant $k$-NN queries. Note that each $k$-NN query has only $k$ correct answers. Hence, (2) becomes

$$
f^{-}(t)=\frac{e^{-}(t)}{k}
$$

which means that at any time $t$, the number of false negatives $\left(e^{-}(t)\right)$ cannot exceed $k$. Moreover, the number of correct objects in the answer returned to the user, i.e., $|A(t)|-e^{+}(t)$, must not be larger than $k$. Equivalently, $1-\frac{e^{+}(t)}{|A(t)|} \leq \frac{k}{|A(t)|}$. Since $\frac{e^{+}(t)}{|A(t)|} \leq \epsilon^{+}(1)$, we get

$$
\begin{gathered}
1-\epsilon^{+} \leq 1-\frac{e^{+}(t)}{|A(t)|} \leq \frac{k}{|A(t)|}, \\
|A(t)| \leq \frac{k}{1-\epsilon^{+}}
\end{gathered}
$$

$$
|A(t)| \leq 2 k .
$$

Equation (8) is obtained by assuming that $\epsilon^{+}<0.5$. Hence, the size of the answer set may not be equal to $k$. For example, if the 10 nearest neighbors are queried with a fraction-based tolerance $\epsilon^{+}=0.1,11$ streams can be returned, where at most one of them is not correct. (That is, all correct ones are returned.) In fact, the answer set size can be controlled by $\epsilon^{+}$and is upper bounded by $2 k$. Finally, since the true answer size is always $k$, we have

$$
\begin{gathered}
|A(t)| \geq k\left(1-\epsilon^{-}\right), \\
|A(t)| \geq \frac{k}{2},
\end{gathered}
$$

when $\epsilon^{-}$is less than 0.5 . Hence, a $k$-NN query answer has a size between $\frac{k}{2}$ and $2 k$. This property will be used in the design of our protocols.

\subsection{Maintaining Query Correctness}

Our protocols translate tolerance constraints into filter constraints installed in the data stream sources. As long as the data value of a stream does not violate the filter constraint, no update is sent from the stream source to the server. When it is necessary that an update be sent to the server, the server may need to reconfigure the filter constraints. We call such reconfiguration constraint resolution. Similar to [4], there are two correctness requirements for our protocols:

Correctness requirement 1 . At every point in time, if no resolution is required, then the results of all running 
continuous queries remain valid within their tolerance constraints.

Correctness requirement 2. Immediately after filter resolution is completed, the tolerance of a query is satisfied assuming that stream values do not change during resolution.

Next, let us study how fraction-based tolerance can be exploited for nonrank-based queries.

\section{Nonrank-Based Queries}

We now study how to exploit fraction-based tolerance for a range query-a nonrank-based query. Consider a protocol that uses no tolerance: each stream filter is assigned the constraint $[l, u]$ at the beginning. Any violation in a filter has to be reported to the server, and query answers are updated correspondingly. Correctness is guaranteed, since essentially, each filter evaluates the range query on the stream it is responsible for. We call this algorithm zero-tolerance protocol for nonrank-based query (ZT-NRP).

Although ZT-NRP can reduce communication costs, it may generate unnecessary updates. Consider Fig. 1 again, where the maximum allowed fraction of false positives is 0.1. Suppose the black-colored object, previously inside the range $R$, has just moved outside. This triggers an update by ZT-NRP. This is not needed, since the black object is the only false positive out of the 10 answer objects. It can thus be included in the answer.

To solve the above problem, we propose a fractiontolerance protocol for nonrank-based query (or FT-NRP in short). Section 4.1 describes the framework of FT-NRP. Section 4.2 investigates how tolerance can be restored if it is violated (by the arrival of data updates). We propose an enhanced protocol in Section 4.3. Section 4.4 extends FTNRP to handle multiple queries.

\subsection{The FT-NRP Framework}

The FT-NRP protocol ensures that at any time during query execution, no more than a fraction $\epsilon^{+}$of query answers are false positives, and no more than a fraction $\epsilon^{-}$of results are false negatives. As shown in Fig. 5, FT-NRP consists of two phases: Initialization and Maintenance of filter constraints.

Initialization. To ensure that no more than a fraction $\epsilon^{+}$ of the answer set (i.e., $A(t)$ ) can be wrong at any time $t$, the server first captures the states of the streams at time $t_{0}$ (Step 1). Then, $A\left(t_{0}\right)$ and $Y\left(t_{0}\right)$, the set of objects which do not belong to $A\left(t_{0}\right)$, are evaluated (Steps 2 and 3 ). Next, the algorithm broadcasts a filter bound $[l, u]$ to all the stream sources involved (Step 4). A subroutine called calError is invoked in Step 5, which computes the maximum number of false positives $\left(e^{\max +}\right)$ and false negatives $\left(e^{\max -}\right)$ allowed, without violating query correctness (refer to the bottom of Fig. 5). According to (3), $e^{\max +}\left(t_{0}\right)=\left|A\left(t_{0}\right)\right| \cdot \epsilon^{+}$. Also, $e^{\max -}\left(t_{0}\right)=\left|A\left(t_{0}\right)\right| \frac{\epsilon^{-}\left(1-\epsilon^{+}\right)}{1-\epsilon^{-}}$(See the footnote. ${ }^{1}$ )

Next, let $n^{+}(t)$ be the number of stream sources allocated the false positive filter constraint (denoted by $[-\infty, \infty]$ ) at time $t$. Stream sources equipped with these filters do not

1. From (2), we have $\epsilon^{-}=\frac{e^{\max -}\left(t_{0}\right)}{\left|A\left(t_{0}\right)\right|-e^{\max }\left(t_{0}\right)+e^{\max -}\left(t_{0}\right)}$. By substituting $e^{\max +}\left(t_{0}\right)=\epsilon^{+}\left|A\left(t_{0}\right)\right|$ (3) into $\epsilon^{+}$, we get $e^{\max -}\left(t_{0}\right)=\left|A\left(t_{0}\right)\right| \frac{\epsilon^{-}\left(1-\epsilon^{+}\right)}{1-\epsilon^{-}}$.

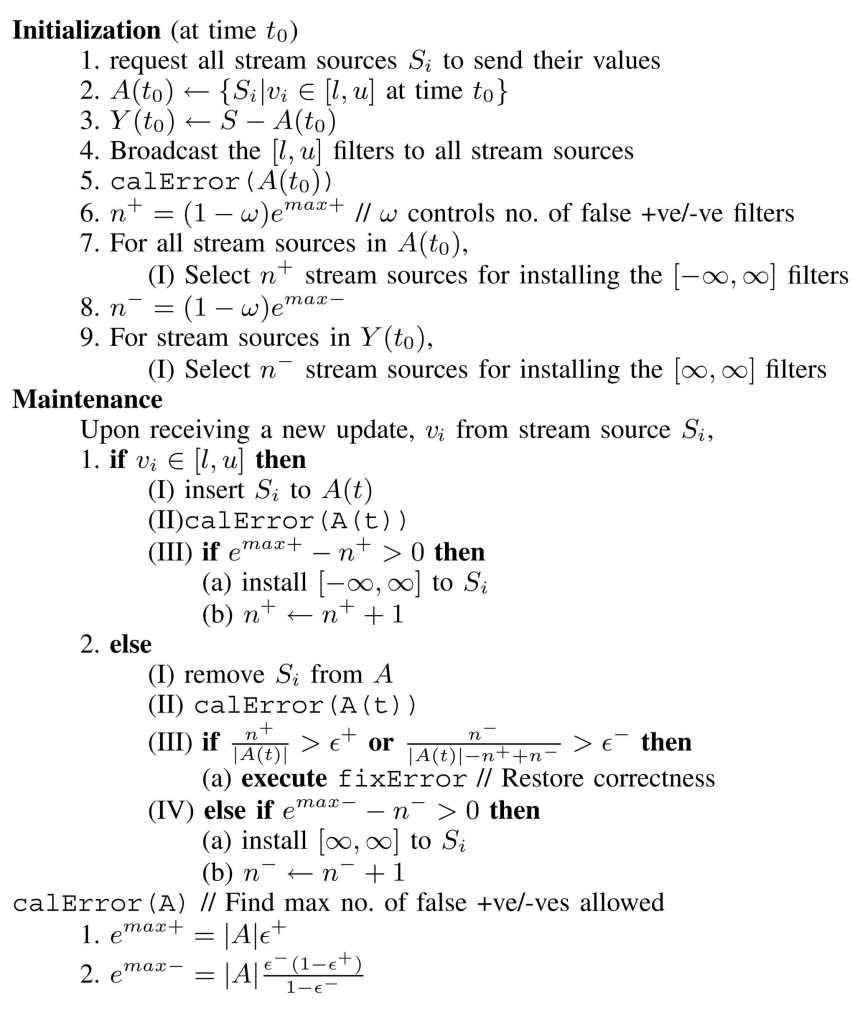

Fig. 5. Maintaining fraction-based tolerance at the server.

report their values at all. Step 6 computes the value of $n^{+}(t)$, which is a fraction $(1-\omega)$ of the maximum false positives allowed (with $\omega \in[0,1]$ ). Out of the $\left|A\left(t_{0}\right)\right|$ answers that satisfy the range query, we assign the $[-\infty, \infty]$ filter constraints to $n^{+}\left(t_{0}\right)$ of them (Step 7). Since $n^{+}(t)$ does not exceed the maximum false positives allowed, if no $[-\infty, \infty]$ stream sources reply, the false positive requirement is met, i.e., $f^{+}(t) \leq \epsilon^{+}$. Moreover, as $n^{+}(t)$ stream sources are "shut down" from emitting updates, the amount of communication is reduced. As illustrated in our experimental results, this approach can also save battery power in a sensor network, since the sensors can be "shut down" and consume less energy than active sensors.

In Step 6, $\omega$ is a system parameter that we call the deployment fraction. Intuitively, $\omega$ controls the number of false positive filters assigned to stream sources in the initialization phase. Why don't we assign these filters to all the $e^{\max +}\left(t_{0}\right)$ and $e^{\max -}\left(t_{0}\right)$ stream sources in Step 7(I)? The main reason is to provide more flexibility in choosing the appropriate number of stream sources to shut down. In particular, we may not know in advance which subset of the streams contributing to the answer $A\left(t_{0}\right)$ would be better associated with the $[-\infty, \infty]$ filters during the initialization phase. If a stream source is wrongly assigned a $[-\infty, \infty]$ filter (e.g., the values generated from that stream are constant), this filter is "wasted" since it does not save any potential update that crosses the $[l, u]$ bounds. On the other hand, for a stream whose value changes around the $[l, u]$ bounds, it is better to associate this stream source with a false positive/negative filter. The maintenance phase, explained next, allocates unused filters generated in the initialization phase to stream 
sources only when they signal to the system that their values have crossed the filter bounds. We term this method, which gradually allocates false positive/negative filters to the stream sources, as incremental deployment. We will also explain later how incremental deployment can be beneficial to FT-NRP. We will also illustrate in the experimental results that it is often better to have a nonzero value of $\omega$, rather than deploying the filters all at once.

False negative tolerance can be exploited in a similar way. Let $n^{-}(t)$ be the number of stream sources allocated the false negative filter constraint (denoted by $[\infty, \infty]$ ) at time $t$. Recall that $\left|Y\left(t_{0}\right)\right|=\left|S-A\left(t_{0}\right)\right|$ streams do not satisfy $Q$. By assigning $[\infty, \infty]$ filters to $n^{-}\left(t_{0}\right)$ of them, these stream sources are "turned off." Since $n^{-}(t)=(1-\omega) e^{\max -}(t)$ (i.e., a fraction of the maximum number of false negative filters), if no data are received from $Y\left(t_{0}\right)$, we are guaranteed at any time $t, f^{-}(t) \leq \epsilon^{-}$(Steps 8-9). Thus, after Initialization Phase, correctness requirement 1 is satisfied. That is, if no update is received at time $t, f^{+}(t) \leq \epsilon^{+}$and $f^{-}(t) \leq \epsilon^{-}$.

Filter selection. To choose which stream sources are assigned the false positive/negative filters in Steps 7(I) and 9(I). We propose two heuristics: 1) random-stream sources are randomly selected and 2) boundary-nearest-only stream sources with values closest to the user-defined query range $[l, u]$ are considered. While random requires only $O(1)$ time, boundary-nearest is more expensive-it requires sorting the distance of the stream value from the query range in $O(n \log (n))$ times. For boundary-nearest, however, objects closer to the query boundary have a higher chance for being assigned the false positive/negative filter constraints. These objects are also the ones that are likely to cross the boundaries and trigger updates. The assignment of filters to these objects can thus increase the chance that updates are dropped by the filters, resulting in better performance.

Maintenance. We now discuss how updates generated from stream sources with $[l, u]$ filters should be managed. Assume that the server receives an updated value $v_{i}$ from $S_{i}$ at time $t_{u}$. Immediately, prior to receiving $v_{i}$, according to correctness 1, the following must hold (using (3) and (4)):

$$
\begin{gathered}
f^{+}(t) \leq \frac{e^{\max +}\left(t_{u}\right)}{\left|A\left(t_{u}\right)\right|} \leq \epsilon^{+}, \\
f^{-}(t) \leq \frac{e^{\max -}\left(t_{u}\right)}{\left|A\left(t_{u}\right)\right|-e^{\max }\left(t_{u}\right)} \leq \epsilon^{-} .
\end{gathered}
$$

Let $t$ be the current time instant with $t \geq t_{u}$. There are two different cases of updates to consider.

Case 1. $v_{i} \in[l, u]$. This means that $S_{i}$, previously not in the result, is now an answer. We handle this by inserting $S_{i}$ into $A\left(t_{u}\right)$ (Step 1(I)). The number of false positives, $e^{+}(t)$, is unchanged. As $|A(t)|$ becomes $\left|A\left(t_{u}\right)\right|+1, f^{+}(t)$ cannot be more than $\frac{e^{\max +}\left(t_{u}\right)}{\left|A\left(t_{u}\right)\right|+1}(3)$, and is less than $\epsilon^{+}(11)$. Since $e^{-}(t)$ is also unchanged, (12) also holds. Thus, correctness 2 is upheld.

The rest of Step 1 uses the false positive filters not deployed in initialization. Specifically, Step 1(II) invokes calError to update $e^{\max +}$. Step 1(III)(a) calculates the quota of $[-\infty, \infty]$ filters that can be allocated to stream sources with $[l, u]$ filters. If this value (equal to $e^{\max +}-n^{+}$) is larger than zero, we assign $[-\infty, \infty]$ to $S_{i}$. We choose $S_{i}$ with the assumption that if $S_{i}$ crosses the $[l, u]$ bound now, it is likely to cross the same bound again later. Thus, any future updates generated from $S_{i}$ can be filtered. Step 1(III)(b) then increments the number of false positive filters allocated.

Case 2. $v_{i} \notin[l, u]$. Stream $S_{i}$ satisfied $Q$ immediately after $[l, u]$ was installed to its filter, but $S_{i}$ is no longer the answer to $Q$ at time $t_{u}$. Step 2(I) removes this "bad answer" from $A\left(t_{u}\right)$ so that the size of $A\left(t_{u}\right)$ is reduced by one. Step 2(II) then updates $e^{\max +}$ and $e^{\max -}$. We check in Step 2(III) whether one of the constraints in (1) and (2) are violated. If so, correctness is violated, and fixError will be invoked (Step 2(III)(a)). Now, let $t_{c}$ be the time when either the false positive or negative fraction (i.e., $f^{+}$and $f^{-}$) attains its maximum value without violating query tolerance. Thus, immediately before fixError is run, we have

$$
|A(t)|=\left|A\left(t_{c}\right)\right|-1 \text {. }
$$

Since fixError can be expensive, its execution should be avoided if possible. Recall that our protocol uses the incremental deployment technique-that is, only some false positive/negative filters are deployed initially. Intuitively, the correctness is "stronger" than required by the tolerance, and thus, reduces the chance for fixError to be called.

If $\mathrm{fixError}$ is not invoked, we check whether there are still some false negative filters not assigned by incremental deployment yet (Step 2(IV)). If so, we assign the $[\infty, \infty]$ filter to stream source $S_{i}$. This assignment does not violate the false negative requirements, since there are false negative filters not allocated. We assume that $S_{i}$ is likely to have values crossing the $[l, u]$ bound in the near future, so that the $[\infty, \infty]$ filter can suppress this update.

\subsection{Error Fixing}

The fixError routine restores query correctness by replacing the false positive/negative filters with $[l, u]$ filters. Recall from Step 2 in Fig. 5 that the violation of correctness requirements is due to the removal of an answer (in Step 2(I)). Let us investigate how fixError tackles this problem. For the purpose of explanation, we classify the streams into four disjoint sets, based on the status of filters, and whether they belong to the query answer:

- $A$ : Stream sources in $A(t)$ with $[l, u]$ filter,

- $B$ : Stream sources in $A(t)$ with $[-\infty, \infty]$ filter,

- $C$ : Stream sources not in answer set $(S-A(t))$ but with $[l, u]$ filter, where $S$ is the set of all stream sources, and

- $D$ : Stream sources in $S-A(t)$ with $[\infty, \infty]$ filter.

Notice that $B$ is the set of stream sources installed with false positive filters, and $D$ contains all stream sources with false negative filters. The legend on the top of Fig. 6 illustrates these four sets of stream sources. The shaded boxes represent sets of stream sources with the $[l, u]$ filters, while the white ones depict those with $[-\infty, \infty]$ or $[\infty, \infty]$ filters.

Recall that fixError is invoked because a stream $S_{i}$ returns an update which is outside the $[l, u]$ bound. As shown in Fig. 7, when $n^{+}>0$, a stream source $S_{y}$ with a false positive filter (i.e., Set $B$ ) is requested to send its value (Step 1(I)). There are two cases, depending on whether $v_{y} \in[l, u]$. 


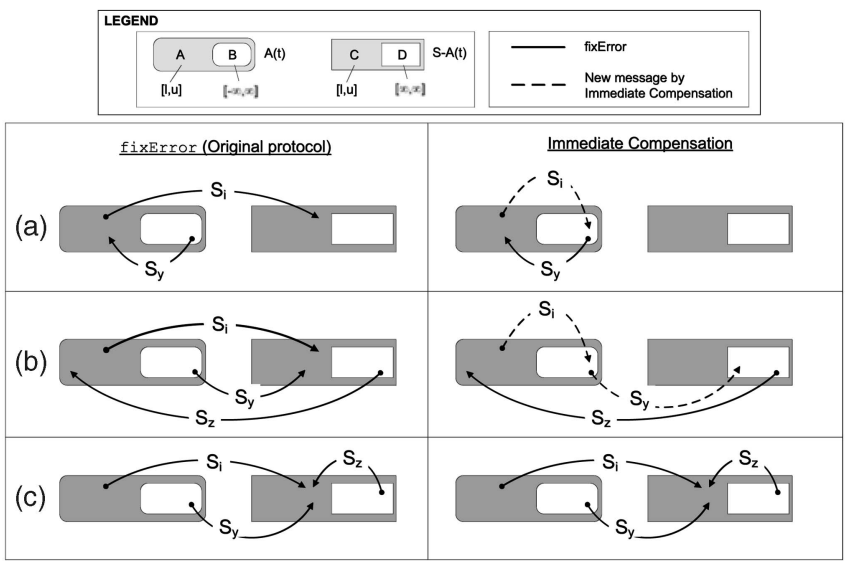

Fig. 6. Cases handled by fixError.

Case 1. $v_{y} \in[l, u]$. This means that $S_{y}$ is currently a true answer. We install a $[l, u]$ filter to $S_{y}$. Hence, $v_{y} \in[l, u]$ when no update is received from it (Step (II)(a)). The left side of Fig. 6a illustrates this, where solid lines represent the change of the sets for $S_{i}$ and $S_{y}$. Notice that as $S_{y}$ has been assigned a false positive filter, $v_{y}$ has already been in $A\left(t_{u}\right)$, and so, $|A(t)|$ remains unchanged (i.e., equal to $\left|A\left(t_{c}\right)\right|-1$ according to (13)). Moreover, $S_{y}$ is no longer a false positive, and so, $e^{+}(t)$ is decremented. Thus, $f^{+}(t)$ is now less than $\frac{e^{\max +}\left(t_{c}\right)-1}{\left|A\left(t_{c}\right)\right|-1}$, which is also less than $f^{+}\left(t_{c}\right)$, meeting the false positive constraint. The false negative constraint is also satisfied: by $(4), f^{-}(t) \leq \frac{e^{\max -}(t)}{|A(t)|-e^{\max +}(t)}$, or $\frac{e^{\max -\left(t_{c}\right)}}{\left(\left|A\left(t_{c}\right)\right|-1\right)-\left(e^{m a x}+\left(t_{c}\right)-1\right)}$, which is less than $\epsilon^{-}$.

Case 2. $v_{y} \notin[l, u] . S_{y}$ is now a true negative, and so, we remove $S_{y}$ from $A(t)$ (13). Then, $|A(t)|$ becomes $\left|A\left(t_{c}\right)\right|-2$ (Step 1(III)). Since $e^{+}(t)$ is also decremented, $f^{+}(t)$ is less than $\frac{e^{\max x}\left(t_{c}\right)-1}{\left|A\left(t_{c}\right)\right|-2}$. As $\epsilon^{+} \leq 0.5, \frac{e^{\max }\left(t_{c}\right)-1}{\left|A\left(t_{c}\right)\right|-2}$ cannot be larger than $\frac{e^{\max x}\left(t_{c}\right)}{\left|A\left(t_{c}\right)\right|}$, and is less than $\epsilon^{+}$.

However, $f^{\max -}(t)$ is at most $\frac{e^{\max -}\left(t_{c}\right)}{\left(\left|A\left(t_{c}\right)\right|-2\right)-\left(e^{\max +}\left(t_{c}\right)-1\right)}$, and can be more than $\epsilon^{-}$. To remedy this, we pick one stream source with a false negative filter (say $S_{z}$ ) from $D$ (Step 2(I)). If $v_{z} \in[l, u]$, we include $S_{z}$ in the answer (Step 2(II)). We also install the $[l, u]$ filter to $S_{z}$ (Step 2 (III)). Now $|A(t)|$ is increased to $\left|A\left(t_{c}\right)\right|-1$, and $f^{-}(t)$ is at most $\frac{e^{\max -\left(t_{c}\right)-1}}{\left(\left|A\left(t_{c}\right)\right|-1\right)-\left(e^{\left.\max +\left(t_{c}\right)-1\right)}\right.}$, which is smaller than $\epsilon^{-}$. Further, $f^{+}(t)$ is at most $\frac{e^{m a x+}\left(t_{c}\right)-1}{\mid A\left(t_{c}\right)-1}$, which is less than $\epsilon^{+}$. Thus, correctness 2 is met, as shown by the solid lines in Fig. 6b.

On the other hand, if $v_{z} \notin[l, u],|A(t)|$ and $e^{+}(t)$ remain unchanged, and thus, the false positive constraint is still satisfied. Since $e^{-}(t)$ is at most $e^{\max -}\left(t_{c}\right)-1, f^{-}(t)$ is at most $\frac{e^{\max }\left(t_{c}\right)-1}{\left.A\left(t_{c}\right) \mid-2\right)-e^{\max +}\left(t_{c}\right)}$, which is smaller than $\epsilon^{-}$because $\epsilon^{-} \leq 0.5$. By assigning the constraint $[l, u]$ to the filter of $S_{z}$, correctness 2 is also met. This case is shown in Fig. 6c.

Notice that fixError involves the sending of three messages in the worst case. It also reduces the number of false positive/negative filters. By preserving some of these filters in the initialization phase and deploying them carefully (i.e., using incremental deployment), the penalty
$S_{y}$ : Set of stream sources with false positive filters $S_{z}$ : Set of stream sources with false negative filters 1. if $n^{+}>0$ then

(I) request value from $S_{y}$ with $[-\infty, \infty]$ constraint

(II) if $v_{y} \in[l, u]$ then

(a) install $[l, u]$ for the filter of $S_{y}$

(b) $n^{+} \leftarrow n^{+}-1$

(c)quit

(III)remove $S_{y}$ from $A(t)$

2. if $n^{-}>0$ then

(I) request value from $S_{z}$ with $[\infty, \infty]$ constraint

(II) if $v_{z} \in[l, u]$ then insert $S_{z}$ into $A(t)$

(III)install $[l, u]$ for the filter of $S_{z}$

(IV) $n^{-} \leftarrow n^{-}-1$

Fig. 7. The fixterror routine (at the server side).

due to the execution of fixError can be reduced, as shown by our experiments.

\subsection{Immediate Compensation}

A problem of fixError is that the false positive/negative filters deployed to the stream sources will be replaced by the $[l, u]$ filters. This causes more communication, since an $[l, u]$ filter allows updates to be generated. To alleviate this, we introduce the Immediate Compensation, which attempts to maintain the number of false positive/negative filters.

Let us examine Fig. 6a again, where the original fixError protocol (on the left) puts $S_{y}$ into the set $A$ by giving it an $[l, u]$ bound, while $S_{i}$ is removed from the answer, i.e., put in set $C$. As a result, the false positive filter associated with $S_{y}$ is "lost." Our new approach avoids this by treating $S_{i}$ as a false positive instead (shown in dotted lines on the right). This is possible because although $S_{i}$ is no longer a valid answer, we can put it into any of the sets $B$, $C$, and $D$. More importantly, by putting $S_{i}$ into set $B$ and $S_{y}$ into set $A$, the "loss" of $S_{i}$ from the answer is immediately compensated by the presence of $S_{y}$. Thus, correctness is restored, and the number of false positive filters remain the same as before.

The same principle can be applied to the scenario in Fig. 6b, where $S_{y}$ is not a query answer. Instead of putting $S_{i}$ into $B$ and $S_{y}$ into $C$ (as in the original fixerror), we can do the following (shown in dotted lines):

1. Put $S_{i}$ into $B$ by associating $S_{i}$ with the $[-\infty, \infty]$ filter.

2. Put $S_{y}$ into $D$ by associating $S_{y}$ with the $[\infty, \infty]$ filter.

From Fig. 6b, $S_{y}$ is replaced by $S_{i}$. Also, the displacement of $S_{z}$ from $D$ is compensated by the arrival of $S_{y}$. Thus, the sizes of all the four sets remain the same, and correctness is fixed. Also, no false positive or false negative filters are compromised after the fixing procedure.

To conclude, the new fixError can retain the false positive/negative filters in cases Figs. $6 a$ and $6 \mathrm{~b}$, but not in Fig. 6c. Thus, in most situations, the performance of the protocol will not deteriorate due to error fixing. The extra cost of immediate compensation compared to the old fixError is that a message is sent to $S_{i}$, for converting its filter from $[l, u]$ to $[-\infty, \infty]$. Our experiments show that this is worthwhile. 


\subsection{Multiple Queries}

Our single-query protocol can be extended to handle situations where more than one continuous query are executed at the same time. First, we apply the initialization step of FT-NRP to each query involved. The filter bounds computed for each query are sent to the stream sources. If there are $m$ concurrent queries, each stream source will be associated with $m$ filter bounds. Second, during maintenance, the value of a stream is checked against the $m$ filter bounds. The new value will only be sent to the server if it violates any of these bounds. Since the update is only sent once even if the value crosses the boundaries of one or more filter bounds, some communication overhead can be saved.

\section{Rank-Based Queries}

A $k$-NN query can be viewed as a range query: if we know the bound $R$ that encloses the $k$ th nearest neighbor of the query point $q$, then any objects with values located within $R$ will be an answer to the $k$-NN query.

We can use this idea to design a filter scheme for $k$-NN query (with zero tolerance). We call this protocol ZT-RP. During initialization, it computes $R$, and then, distributes $R$ to all the stream filters. If no responses are received from the streams, the server is assured that all $k$ objects are within $R$, and they are still the $k$ nearest neighbors of $q$. Since no error is allowed, if any object enters or leaves $R$, we have to recompute $R$ so that $R$ still encloses the $k$ nearest objects. In addition, the new $R$ has to be announced to every stream.

The main drawback of this simple protocol is that it is sensitive to an object's value crossing $R$. When this happens, $R$ has to be recomputed and announced to every stream! Let us investigate how this problem can be alleviated.

\subsection{Using FT-NRP for $k$-NN Query}

We just discussed how to view a $k$-NN query as a range query for the purpose of constraint deployment. Recall that the definition of fraction-based tolerance is the same for $k$-NN query and range query. To develop a fraction-based tolerance protocol for a $k$-NN query, one may consider transforming a $k$-NN query to a range query, and then, directly apply FT-NRP. Unfortunately, this is incorrect. As we show shortly, the $\epsilon^{+}$and $\epsilon^{-}$parameters of a $k$-NN have to be first converted to two other values.

Specifically, let $\rho^{+}$and $\rho^{-}$be the maximum false positive and negative tolerance values used by FT-NRP, in order to answer a $k$-NN query with tolerance $\epsilon^{+}$and $\epsilon^{-}$. Let $R$ be the smallest region that initially bounds the $k$ th-ranked object, and thus, contains $k$ objects. Similar to the initialization of FT-NRP, for objects with values in $R$, we assign false positive filters to $k \rho^{+}$stream sources; for stream sources with values outside $R$, we apply false negative filters to $k \rho^{-}$stream sources. Other stream sources use $R$ as the filter bounds. Let us examine how $\rho^{+}$and $\rho^{-}$should be set.

Meeting false positive requirement. Suppose $R$ encloses the $k$ nearest objects of $q$. Let $S_{1}$ be part of the answer set, and $v_{1}^{\prime} \in R$ is the value of $S_{1}$ last reported to the server. Hence, $S_{1}$ is one of the $k$ nearest neighbors. If $S_{1}$ is associated with a false positive filter, the new value of $S_{1}$, i.e., $v_{1}$, may not be located within $R$. Consider the situation shown in Fig. 8. Suppose there exists a stream $S_{2}$ such that $v_{1}<v_{2}$. Then, $S_{1}$ is no longer a correct answer, since $S_{2}$ now ranks higher and it pushes the rank of $S_{1}$ to $k+1$. Therefore,

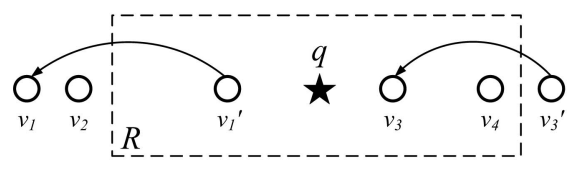

Fig. 8. False positives and false negatives for a $k$-NN query.

$S_{1}$ becomes a false positive. Since at most $|A(t)| \rho^{+}$stream sources are assigned with false positive filters, at most $|A(t)| \rho^{+}$false positives may be produced in this way.

Another kind of false positive is caused by false negative filters. Suppose $S_{4}$, being ranked $k$ th and lies within $R$, is an answer. Also assume that $S_{3}$ has a false negative filter, whose last reported value $v_{3}^{\prime}$ is outside $R$. Fig. 8 shows that when the new value of $S_{3}$, i.e., $v_{3}$, is inside $R$, the rank of $S_{3}$ is $k$ or higher. The rank of $S_{4}$ is demoted to $k+1$, and thus, $S_{4}$ becomes a false positive. Since false negative filters can be assigned to at most $k \rho^{-}$stream sources (5), at most $k \rho^{-}$false positives are created.

The sum of the false positives generated by these two scenarios is $|A(t)| \rho^{+}+k \rho^{-}$, where $|A(t)|<\frac{k}{1-\epsilon^{+}}$(7). Also, there cannot be more than $|A(t)| \epsilon^{+}$false positives, with a minimum value of $k\left(1-\epsilon^{-}\right) \epsilon^{+}(9)$. Therefore,

$$
\rho^{-} \leq \frac{\rho^{+}}{\epsilon^{+}-1}+\left(1-\epsilon^{-}\right) \epsilon^{+} .
$$

Meeting false negative requirement. Again, there are two types of false negatives for a $k$-NN query. As shown in Fig. 8, the first type of false negatives is caused by streams like $S_{3}$, whose last reported value $v_{3}^{\prime}$ is not within $R$, and is assigned with false negative filters. Later, its new value $v_{3}$ is within $R$ and its rank is raised to $k$ or higher. The server does not know this, and so, $S_{3}$ is a false negative. The number of false negatives is at most $k \rho^{-}$, the maximum number of false negative filters. The second type is caused by stream sources with false positive filters like $S_{1}$. Again, $S_{1}$ was among the top- $k$ objects since its last reported value $v_{1}^{\prime}$ is within $R$. However, its new value $v_{1}$ is less than $v_{2}$, so $S_{2}$ ranks $k$ or higher (without notifying the server). The maximum number of this kind of false negatives is thus $|A(t)| \rho^{+}$, the maximum number of false positive filters. Since the maximum number of false negatives for $k$-NN query is given by $k \epsilon^{-}$, the sum of the two kinds of false negatives $k \rho^{-}$and $|A(t)| \rho^{+}$must be less than $k \epsilon^{-}$. Equation (7) simplifies this to

$$
\rho^{-} \leq \frac{\rho^{+}}{\epsilon^{+}-1}+\epsilon^{-} .
$$

Guaranteeing correctness. To make sure that both false positives and false negatives are met, we combine (14) and (15) so that the following is achieved:

$$
\rho^{-} \leq \frac{\rho^{+}}{\epsilon^{+}-1}+\min \left(\left(1-\epsilon^{-}\right) \epsilon^{+}, \epsilon^{-}\right) .
$$

Essentially, given the tolerance $\epsilon^{+}$and $\epsilon^{-}$, the values of $\rho^{+}$ and $\rho^{-}$must be configured to satisfy (16). To maximize the benefit, $\rho^{+}$and $\rho^{-}$should set as

$$
\rho^{-}=\frac{\rho^{+}}{\epsilon^{+}-1}+\min \left(\left(1-\epsilon^{-}\right) \epsilon^{+}, \epsilon^{-}\right) .
$$




\subsection{Fraction-Based Tolerant $k$-NN Query}

Once the values of $\rho^{+}$and $\rho^{-}$are correctly set, we can extend FT-NRP to exploit the fraction-based tolerance of $k$-NN queries. The corresponding protocol, called FT-RP, differs from FT-NRP in two aspects:

1. Unlike a range query with a fixed bound $[l, u]$, the "range" of $k$-NN query is defined by $R$-the tightest bound that contains the $k$ th nearest neighbor. Thus, FT-RP first finds $R$ before running the initialization phase of FT-NRP. Notice that the filter constraint $R$ so calculated will not be changed even when $R$ contains more or less than $k$ objects-except when the conditions described next are met. Essentially, we use $R$ only as an estimate of the $k$ nearest neighbors.

2. A requirement for the answer $A(t)$ of a rank-based query is that $k\left(1-\epsilon^{-}\right) \leq|A(t)| \leq \frac{k}{1-\epsilon^{+}}((7)$ and (9)). Initially, $|A(t)|=k$, but as time goes by, the number of items in $A(t)$ increases (decreases) when an object enters (exits) $R$. Intuitively, when $|A(t)|>\frac{k}{1-\epsilon^{+}}$, there are too many objects in $R$, i.e., $R$ is "too loose." Similarly, when $|A(t)| \leq k\left(1-\epsilon^{-}\right)$, too few objects are in $R$, i.e., $R$ is "too tight." In either case, $R$ is no longer an appropriate bound. We need to find a bound that encloses the new $k$-nearest neighbors.

Thus, the advantage of FT-RP over ZT-RP is that it does not recompute and broadcast $R$ when an object enters or leaves $R$, but only when $A(t)$ drops below $k\left(1-\epsilon^{-}\right)$or exceeds $\frac{k}{1-\epsilon^{+}}$.

\section{Performance Evaluation}

We now examine the performance of our protocols. Sections 6.1,6.2, and 6.3 analyze the computation, communication, and power consumption of ZT-NRP and FT-NRP, respectively. Section 6.4 presents the experimental setup, and Section 6.5 discusses the results.

\subsection{Computational Complexity}

Let us first consider the computation cost of the processing server in ZT-NRP. In the beginning, ZT-NRP just needs to send the $[l, u]$ bound to the stream sources. Any update received from the stream sources will be used to refresh the query answer. Thus, both initialization and maintenance need $O(1)$ time.

For FT-NRP, during initialization, Steps $1-3$ require a complexity of $O(n)$. Steps $4,5,6$, and 8 cost $O(1)$. If random selection is used, Steps 7 and 9 require a total of $O\left((1-\omega)\left(e^{\max +}+e^{\max -}\right)\right)$. Thus, initialization requires $O(n)$. If nearest-boundary selection is used, an additional sorting cost of $O(n \log (n))$ is required, and initialization costs $O(n \log (n))$. For maintenance, notice that the complexity of fixError is $O(1)$ (Fig. 7). Thus, the maintenance phase requires only $O(1)$ for every update received by the server.

\subsection{Communication Cost Model}

Our distributed communication model is similar to the one described in [26], where a server can broadcast its messages to the stream sources. These messages are classified into three categories.
- Broadcast $\operatorname{cost}\left(C_{b}\right)$ for broadcasting a message from the server to all stream sources.

- Downlink $\operatorname{cost}\left(C_{d}\right)$ for sending a downlink message from the server to a stream source.

- Uplink $\operatorname{cost}\left(C_{u}\right)$ for sending an uplink message from a stream source to the server.

This cost model can be used to characterize the type of resources studied. For example, the cost can be the network bandwidth or energy consumed by substituting appropriate values. In Section 6.3, we discuss how power costs can be considered based on this model.

For ZT-NRP, the initialization process needs a cost of $C_{b}$ for broadcasting the $[l, u]$ bound to all stream sources. During maintenance, a cost of $C_{u}$ is needed for a stream source to report its value. As for FT-NRP, during initialization, all stream sources send their initial values in response to the broadcast request from the server (Step 1). Therefore, a cost of $C_{b}+n C_{u}$ is needed. Then, the server broadcasts a message which consists of the $[l, u]$ bound to every stream source, with a cost of $C_{b}$. After the initial set of false positives and negatives is determined, the FT-NRP needs a cost of $(1-\omega)\left(e^{\max +}+e^{\max -}\right) C_{d}$ to deploy the false positive and false negative filters to stream sources (Steps 6-9). Thus, the communication cost during initialization is

$$
2 C_{b}+n C_{u}+(1-\omega)\left(e^{\max +}+e^{\max -}\right) C_{d},
$$

i.e., a complexity of $O(n)$.

During maintenance, each update from a stream source will incur a cost of $C_{u}$. If resolution takes place, it requires a cost of $C_{d}+C_{u}$ for requesting a value from a false positive stream source $\left(S_{y}\right)$. If the protocol further requires a false negative stream source $S_{z}$ be interrogated, a cost of $\left(C_{d}+\right.$ $C_{u}$ ) is needed. Therefore, the cost of maintenance is at most

$$
3 C_{u}+2 C_{d} .
$$

For immediate compensation, an extra message for updating the filter of $S_{i}$ may be needed, and so in the worst case, its cost is

$$
3\left(C_{u}+C_{d}\right)
$$

Thus, maintenance of FT-NRP requires a communication cost of $O(1)$. Although immediate compensation is more expensive, our experiments show that this extra cost is worth paying. If we substitute the values of $C_{b}, C_{d}$, and $C_{u}$ by 1 , then the above analysis will yield the total number of messages used. Next, we study the energy consumption costs.

\subsection{Power Consumption of Sensor Networks}

In many data stream applications, battery power is a precious resource. For example, in wireless sensor networks (WSNs), sensors are only equipped with a limited power source (e.g., $0.5 \mathrm{Ah}, 1.2 \mathrm{~V}$ ) [2]. Therefore, it is important to preserve energy resources for the sensors in these systems.

Let us now study the energy requirements of sensors in FT-NRP and ZT-NRP. Assume that each sensor is a data stream source, each of which can be installed with a filter. According to [24], there are two types of energy consumption due to data communication. 
- Transmission power $\left(P_{t}\right)$ for sending an uplink message to the server.

- Reception power $\left(P_{r}\right)$ for receiving a downlink message from the server at the stream source.

The amount of energy consumed due to communication can now be deduced by using the communication cost model described in the previous section. In particular, the broadcast cost $C_{b}$ becomes $n P_{r}$, since all the $n$ sensors will receive a message from the server. We also equate the downlink cost $\left(C_{d}\right)$ to $P_{r}$, and the uplink cost $\left(C_{u}\right)$ to $P_{t}$. By substituting these values into the results obtained in Section 6.2, we can see that ZT-NRP requires an initialization and maintenance cost of $n P_{r}$ and $P_{t}$, respectively. For FT-NRP, we have:

- Initialization: $n P_{t}+\left(2 n+(1-\omega)\left(e^{\max +}+e^{\max -}\right)\right) P_{r}$.

- Maintenance (without immediate compensation): $3 P_{t}+2 P_{r}$.

- Maintenance (with immediate compensation): $3\left(P_{t}+P_{r}\right)$.

In addition to communication, a sensor needs to collect information from the external environment. We use sensing power $\left(P_{s}\right)$ to denote the amount of energy required for a sensor to acquire a data value (e.g., temperature, location). We assume that unless a sensor is installed with a false positive/negative filter, it collects data at a fixed periodicity (or duty cycle). At any instant of time during maintenance, for ZT-NRP, there are $n$ stream sources installed with $[l, u]$ filters. Thus, the total amount of sensing power consumed in the maintenance phase is $n P_{s}$. On the other hand, FT-NRP only has $\left(|A(t)|-n^{+}\right)+\left(|S-A(t)|-n^{-}\right)$stream sources installed with $[l, u]$ filters, and so, the sensing power required is $\left(\left(|A(t)|-n^{+}\right)+\left(|S-A(t)|-n^{-}\right)\right) \times P_{s}$. The higher the number of false positive filters $\left(n^{+}\right)$or false negative filters $\left(n^{-}\right)$, the more sensing energy is saved.

In the rest of this section, we present the experimental results for the network bandwidth and power consumption performance of our protocols.

\subsection{Experimental Setup}

We use CSIM 19 [33] to simulate the environment illustrated in Fig. 3. We test the performance based on both real and synthetic data.

For real data, we choose the TCP traces described in [23]. Our experiment models a remote network monitoring application, where a central console is used to monitor a network composed of 800 subnets. The data set contains 30 days of wide-area traces of TCP connections, capturing 606,497 connections. Each subnet represents a stream source. The "number of bytes sent" field in each packet trace is used as a data value. We assume that an agent software that implements our filters is installed at each subnet router. We consider two kinds of queries: a top- $k$ query and a range query. These two queries are important to the network monitoring domain. Specifically, the heavy hitter analysis is often used to continuously discover an IP-source (called "heavy hitter") that delivers the largest number of packets to the monitored network, which is also likely involved in DOS attacks [5], [15]. If the user only needs to know the top- $k$ heavy hitters, then this problem can be modeled as a top- $k$ query, which reports continuously the subnets with the $k$ highest traffic volume [4]. The range query can be used to classify subnets with different ranges of traffic volume. It also captures IP sources that may participate in a DOS attack, if the amount of traffic is identified to be within a "dangerous" range. Here, we assume $k=60$, and a query range of [200, 350].

We also perform testing on synthetic data, where 5,000 stream sources are generated. The time between each data item generated follows an exponential distribution with a mean of 20 time units. The values of a data stream item is uniformly distributed in $[0,1,000]$. When a new data value is generated, its difference from the previous value follows a normal distribution with a mean of zero and a variance of 60 units. For range queries, we assume that the default query range is $[400,600]$. For rank-based queries, we simulate the $k$-NN queries with $k$ equal to 60 . These parameter values are tuned in such a way that interesting trends can be observed and compared with the real data set. We also examine the effect of varying the value distribution of the synthetic data set.

For the FT-NRP protocol, the default value of $\omega$ is zero, and immediate compensation is not used. These are the same parameter settings for the experiments used in our previous paper [7].

The two metrics used to measure the protocol performance are: 1) the total number of messages generated and 2 ) the amount of energy consumed, based on the cost model described in Sections 6.2 and 6.3. For the parameters of power consumption, we adopt the specifications used by an MICA2 mote [9]: the operation voltage of each sensor is $3.6 \mathrm{~V}$, the transmission power $P_{t}$ is $77.4 \mathrm{~mJ}$ (at a distance of $+10 \mathrm{dBm}$ ), the reception power is $25.2 \mathrm{~mJ}$, and the sensing power $P_{s}$ is $2.52 \mathrm{~mJ}$. The sensing frequency is once every 5,000 time units.

\subsection{Results}

We describe the simulation results for range queries, a nonrank-based query, in Section 6.5.1. Section 6.5.2 presents the results for $k$-NN query, a rank-based query.

\subsubsection{Nonrank-Based Queries}

We examine how well the FT-NRP protocol exploits the saving in messages and energy consumption for range queries. Fig. 9a shows that the number of messages decreases as $\epsilon^{+}$and $\epsilon^{-}$increase. For example, at $\epsilon^{+}=$ $\epsilon^{-}=0.1$, the improvement over ZT-NRP (which does not exploit tolerance) is 2.65 percent. Hence, FT-NRP performs better than ZT-NRP. Fig. 9b shows a similar behavior for energy consumption: 3.49 percent saving over ZT-NRP is achieved at $\epsilon^{+}=\epsilon^{-}=0.1$; and about 38 percent of power is saved at $\epsilon^{+}=\epsilon^{-}=0.5$.

For synthetic data, we examine FT-NRP under different values of $\epsilon^{+}$and $\epsilon^{-}$. Figs. 9c and 9d show that FT-NRP exploits tolerance on both messages and energy saving effectively similar to the real data. For example, at $\epsilon^{+}=\epsilon^{-}=0.1$, the message and energy savings are 2.25 and 2.22 percent, respectively. The relatively small improvement at small tolerance values (e.g., $\epsilon^{+}=\epsilon^{-}=0.1$ ) is due to the fact that the answer set is quite small (20 percent of stream 


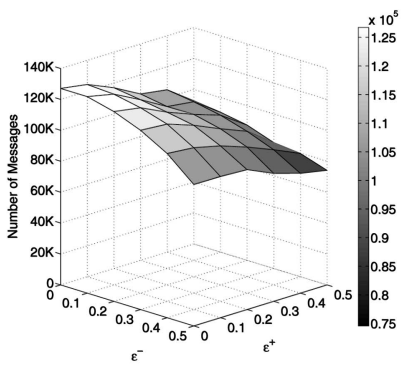

(a)

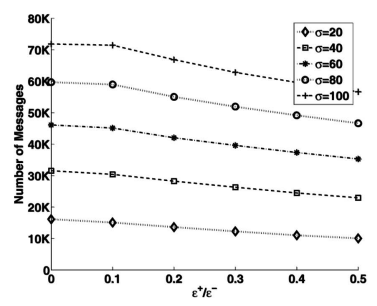

(e)

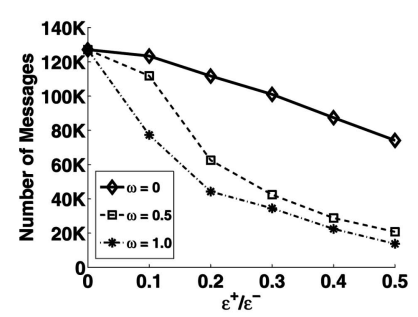

(i)

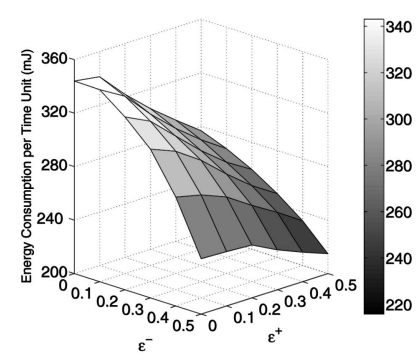

(b)

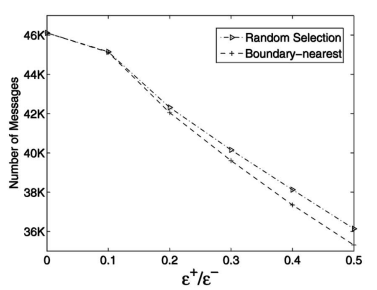

(f)

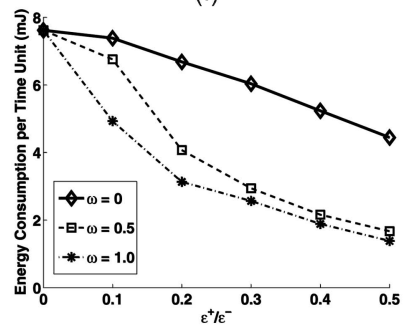

(j)

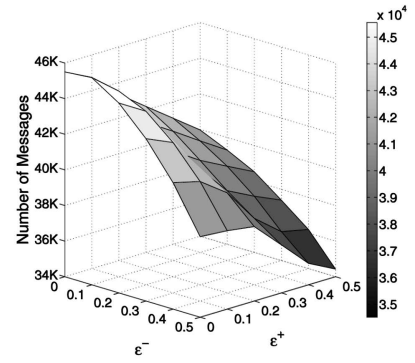

(c)

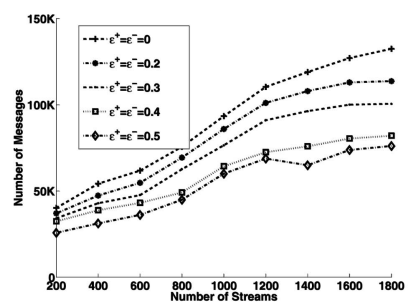

(g)

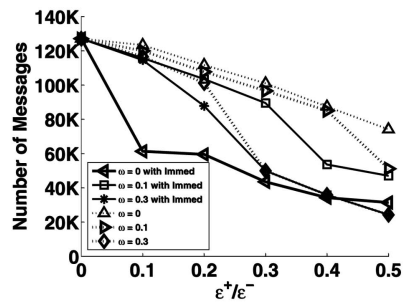

(k)

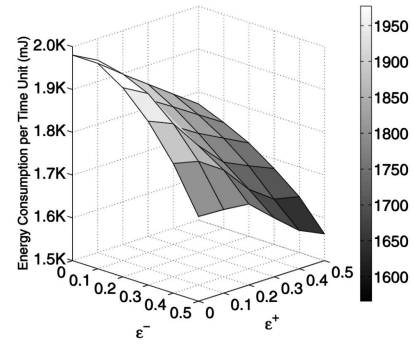

(d)

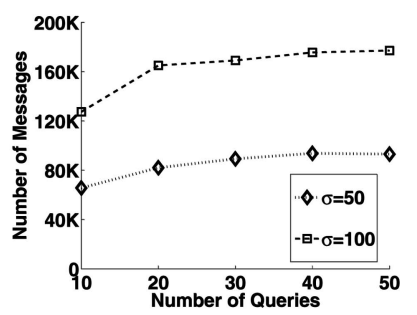

(h)

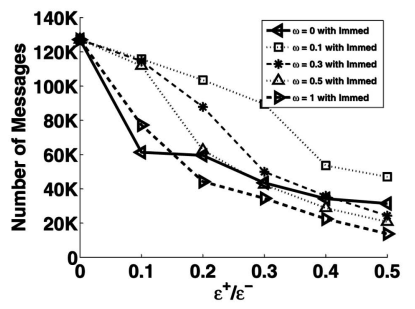

(I)

Fig. 9. Experiments on FT-NRP. (a) \# messages (real). (b) Energy (real). (c) \# messages (synthetic). (d) Energy (synthetic). (e) Data fluctuation. (f) Selection heuristics. (g) Scalability. (h) Multiple queries. (i) Incremental deployment (\# messages). (j) Incremental deployment (energy). (k) Immediate compensation. (I) Immediate compensation and $\omega$.

sources), and so, only a small number of false positive/ negative filters can be used. The overhead of maintaining these filters thus affects the performance of the protocol.

Data fluctuation. Fig. 9e illustrates the effect of data fluctuation (i.e., the amount of difference between two adjacent values in a stream) on FT-NRP, for synthetic data. We test the cases when $\epsilon^{+}=\epsilon^{-}$, with different values of standard deviation $(\sigma)$. For all values of $\sigma$ tested, the performance improves with larger values of $\epsilon^{+}$and $\epsilon^{-}$. Another observation is that as $\sigma$ increases, FT-NRP yields more messages. When a data value changes more abruptly, it has a higher chance of violating the filter bound constraint and generating an update.

Selection heuristics. We explore how FT-NRP is affected by the assignment of false positive/negative filters during initialization. Specifically, we compare the performance of two heuristics: random and boundarynearest. Fig. 9f shows that boundary-nearest is only slightly better than random. The difference is small because the assumption that values closer to the query boundary are more likely for its future values to cross the boundary again may not hold. For example, an object can be moving away from the boundary, and so, even if it is close to the boundary during initialization, it is not necessarily worth to assign a false positive/negative filter to its corresponding stream source. As we will illustrate, the use of incremental deployment-assign a fraction of $1-\omega$ filters to the stream sources, and only allocate a filter to the stream source when it generates an update-often results in a better performance.

Let us now focus on real data.

Scalability with data and queries. We also test the effect of the number of stream sources and queries on FT-NRP. Fig. $9 \mathrm{~g}$ shows that FT-NRP scales well with the number of stream sources, under different values of $\epsilon^{+}$and $\epsilon^{-}$. We further investigate the performance of our protocols in handling multiple queries, as discussed in Section 4.4. We consider a varying number of queries under different distributions. These queries are executed at the same time. The centers of the query ranges follow a normal distribution with a standard deviation $(\sigma)$. As shown in Fig. 9h, the protocols, in general, scale well over a large range of number of queries. The number of messages does not increase linearly with the number of queries. This is because individual updates can be shared by more queries as the number of queries increases. Consider, for instance, two range queries that have a high degree of overlap. Then, the crossing of boundary for one query will likely co-occurs with the crossing of the other query's boundary. Hence, only one update is necessary for both queries. With a similar argument, we can see that the protocol performs better when the distribution of the queries is denser (i.e., decreases from $\sigma=100$ to $\sigma=50$ ), because each update can be shared by more queries. 


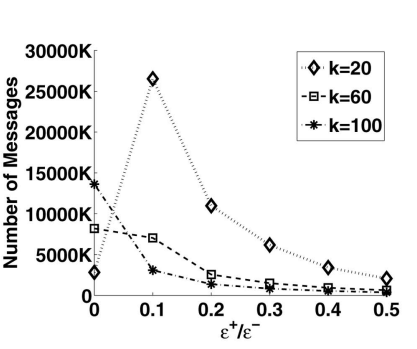

(a)

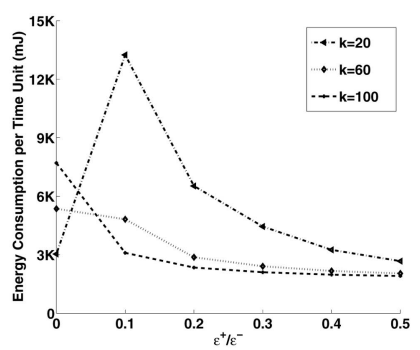

(b)

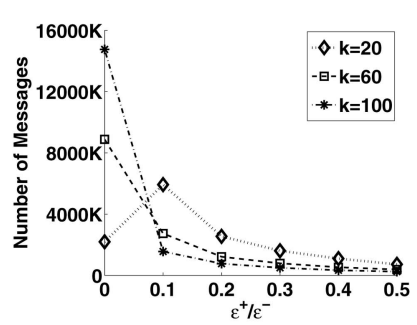

(c)

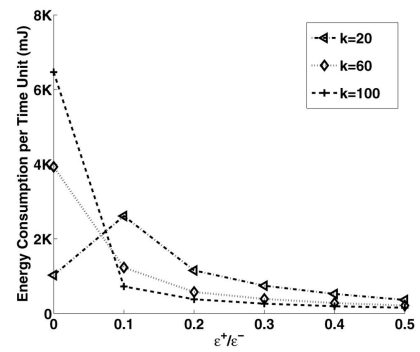

(d)

Fig. 10. Experiments on FT-RP. (a) \# messages (real). (b) Energy (real). (c) \# messages (synthetic). (d) Energy (synthetic).

Incremental deployment. Fig. 9i illustrates the effect of $\omega$ on the number of messages. Recall that the larger the value of $\omega$, the lower the number of stream sources is assigned with false positive/negative filters in the initialization phase. These filters are only assigned in the maintenance phase, when their values cross the query boundaries. We can see that the performance improves with a larger value of $\omega$. There are two reasons. First, before the initialization phase, we may not know exactly which stream sources are worthy to be installed with the false positive/negative filters. If a stream source is assigned with a false positive filter but its value actually has little chance of crossing the boundary, then this filter is wasted. By delaying the filter assignment until a stream's value has crossed the query boundary, the filter is allocated to a stream source which is more "active" (in crossing the query boundary), so that any other updates generated by this stream source are dropped. Second, observe that fixError will be executed in Step 2(III)(a) if one of the two conditions is violated. If $n^{+}$and $n^{-}$are smaller than the maximum values allowed (correspondingly $e^{\max +}$ and $\left.e^{m i n-}\right)$, then fixError will not be executed. If $\omega$ is nonzero, then the initial values of $n^{+}$and $n^{-}$will be less than the maximum values allowed, and so, fixerror, which is a costly operation, will be less likely to be executed upon the arrival of updates. We can observe similar results for energy saving, as shown in Fig. 9j.

Immediate compensation. In this experiment, we examine the use of immediate compensation in fixError. Fig. 9k shows the number of messages required, for three values of $\omega(0,0.1$ and 0.3$)$. We can see that for all the values of $\omega$ shown, immediate compensation provides better performance than if it is not used. This is because the fixError in [7] replaces some false positive/negative filters with $[l, u]$ filters. Hence, fewer updates can be filtered after its execution. Immediate compensation, on the other hand, maintains the number of false positive/negative filters in most cases. Therefore, it provides a better performance than the original protocol.

We also note that the effect of immediate compensation is more profound at smaller values of $\omega$. This is because at a large $\omega$, more false positive/negative filters are preserved for future use, and it is less likely for fixError to be invoked (fixError is only executed if all the false positive/negative filters have been assigned). In fact, although not shown in the graph, when $\omega$ is larger than 0.5 , immediate compensation almost yields no benefit. Thus, immediate compensation is more useful for small $\omega$ values.
In Fig. 91, we show the effect of immediate compensation for different $\omega$ values. We can see that except for $\omega=0$, as $\omega$ increases, the performance improves. For $\omega=0$, all the false positive/negative filters have been assigned during initialization, and more stream sources can be allocated with filters in the beginning than other $\omega$ values. If immediate compensation is further used, with $\omega=0$, the protocol could retain the largest number of filters after fixError, and so, it performs well in this experiment.

\subsubsection{Rank-Based Queries}

Next, we examine how FT-RP exploits the fraction-based tolerance for $k$-NN query, a rank-based query. Fig. 10a shows the result under three values of $k: 20,60$, and 100. As we can see, when $k$ is 60 or 100 , the number of messages drops significantly with a small increase in tolerance. This is because the bound $R$ for enclosing the $k$ nearest objects is not "tight," and so, objects can cross $R$ without requiring $R$ to be recomputed and sent to the stream sources. With zero tolerance, however, $R$ virtually changes every time an object crosses it. When $k=20$ and $\epsilon^{+}=\epsilon^{-}=0.1$, the protocol yields a high message cost. This is because the number of false positive/negative filters assigned is limited. Therefore, the little benefit of tolerance cannot overcome the high maintenance cost. Thus, FT-RP is not suitable in this situation. For energy consumption, the radio energy incurred by message transmission dominates; thus, as shown in Fig. 10b, the overall energy consumption follows the same behavior as message count.

Figs. 10c and 10d show the performance of the protocols for the synthetic data set. The result is similar to the case of real data. We note that the performance for $k=20$ for synthetic data is better than the real data set. The reason is that the values of the synthetic data are relatively stable (i.e., they crossed the $R$ bound less frequently). Thus, the number of updates generated, as well as the recomputation of $R$, is smaller.

Finally, we examine Incremental Deployment and Immediate Compensation for FT-RP using the real data set. We assume $k=60$. Figs. $11 \mathrm{a}$ and $11 \mathrm{~b}$ show the results of message count and energy consumption, respectively. In contrast to FT-NRP, Incremental Deployment has a relatively small improvement. Recall from (17) that the values of $\rho^{+}$and $\rho^{-}$are usually smaller than the user-specified tolerance (i.e., $\epsilon^{+}$and $\epsilon^{-}$). Thus, the number of false positive/negative filters computed is small, and the performance is relatively insensitive to the value of $\omega$. 


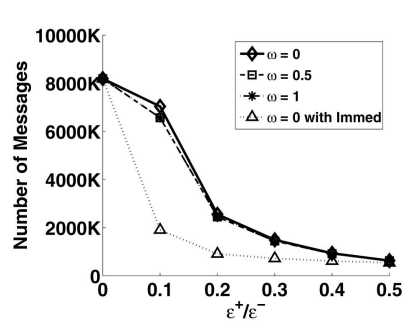

(a)

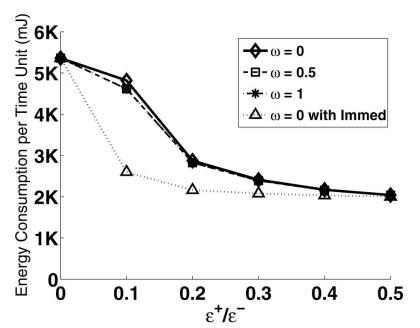

(b)
Fig. 11. Incremental deployment (FT-RP). (a) \# messages (real). (b) Energy (real).

On the other hand, Immediate Compensation performs much better when the tolerance is at $0.1-$ a message saving of 75 percent can be achieved. Due to the smaller number of false positive/negative filters available, fixError has to be executed more frequently. By using Immediate Compensation, the false positive/negative filters are more likely to be retained after the evaluation of fixError. This, in turn, reduces the number of recomputation and deployment of the new $R$ bound, resulting in a better performance.

\section{Conclusions}

In this paper, we developed protocols to improve the performance of data stream management systems. The main idea of these protocols is to translate fraction-based query tolerance to filter bounds, and transmit these bounds to the stream sources. The stream sources can then conditionally drop their updates without affecting query correctness. We designed algorithms that initiate and maintain filter bounds for nonrank- and rank-based queries. We further presented two variations of these protocols, namely, incremental deployment and immediate compensation. Through detailed testing on real and synthetic data, we verified the effectiveness of our protocols in reducing communication and energy costs.

For future work, we will enhance our algorithm to adapt to the addition of stream sources during query execution. We also want to examine how the value distribution across different data streams can be used to design better filter assignment policies. We will address how other data stream optimization algorithms (e.g., synopses construction and load shedding) can employ the notion of fraction-based tolerance. We will also study how this new notion can be used to design filters for other query types (e.g., joins). Another interesting study is to design a new correctness notion that combines the advantages of value-based and nonvalue-based tolerances, and develop filtering protocols accordingly. Such a tolerance could be useful when a user is not only concerned about the number of false positives, but also that the actual values of the false answers are not too far away from the query range. Another interesting question is how our protocols can be applied to sensor-networked environments, where each sensor can be viewed as a stream source. The challenge is to exploit the unique characteristics of sensor networks (e.g., sensors are arranged in a hierarchical manner) in order to further optimize the use of energy and bandwidth resources.

\section{ACKNOWLEDGMENTS}

This work was supported by the Research Grants Council of Hong Kong (GRF Projects 513508 and 513307), the Germany/ HK Joint Research Scheme (Project G_HK013/06), and the University of Hong Kong (Project 200808159002). The authors thank the reviewers for their insightful comments.

\section{REFERENCES}

[1] D. Abadi et al., "The Design of the Borealis Stream Processing Engine," Proc. Second Biennial Conf. Innovative Data Systems Research (CIDR), 2005.

[2] I.F. Akyildiz, W. Su, Y. Sankarasubramaniam, and E. Cayirci, "Wireless Sensor Networks: A Survey," Computer Networks J., vol. 38, no. 4, pp. 393-422, Mar. 2002.

[3] A. Arasu et al., "Characterizing Memory Requirements for Queries over Continuous Data Streams," ACM Trans. Database Systems, vol. 29, no. 1, pp. 162-194, 2004.

[4] B. Babcock and C. Olston, "Distributed Top-k Monitoring," Proc. ACM SIGMOD, 2003.

[5] M. Charikar, K. Chen, and M. Farach-Colton, "Finding Frequent Items in Data Streams," Theoretical Computer Science, vol. 312, pp. 3-15, 2004.

[6] R. Cheng, D. Kalashnikov, and S. Prabhakar, "Evaluating Probabilistic Queries over Imprecise Data," Proc. ACM SIGMOD, 2003.

[7] R. Cheng, B. Kao, S. Prabhakar, A. Kwan, and Y. Tu, "Adaptive Stream Filters for Entity-Based Queries with Non-Value Tolerance," Proc. Int'l Conf. Very Large Data Bases (VLDB), 2005.

[8] Y. Chi, H. Wang, P. Yu, and R. Muntz, "Loadstar: A Load Shedding Scheme for Classifying Data Streams," Proc. SIAM Conf. Data Mining, 2005.

[9] MPR-Mote Processor Radio Board User's Manual. Crossbow, Inc., 2003.

[10] B. Cui et al., "Exploring Bit-Difference for Approximate knn Search in High-Dimensional Databases," Proc. Australasian Database Conf., 2005.

[11] A. Deligiannakis, Y. Kotidis, and N. Roussopoulos, "Hierarchical In-Network Data Aggregation with Quality Guarantees," Proc. Conf. Extending Database Technology (EDBT), 2004.

[12] A. Deshpande, C. Guestrin, S. Madden, J. Hellerstein, and W. Hong, "Model-Driven Data Acquisition in Sensor Networks," Proc. Int'l Conf. Very Large Data Bases (VLDB), 2004.

[13] L. Doherty, B.A. Warneke, B.E. Boser, and K.S.J. Peter, "Energy and Performance Considerations for Smart Dust," Int'l J. Parallel and Distributed Sensor Networks, vol. 4, no. 3, pp. 121-133, 2001.

[14] D. Abadiand et al., "Aurora: A Data Stream Management System," Proc. ACM SIGMOD, 2003.

[15] S. Ganguly, M. Garofalakis, R. Rastogi, and K. Sabnani, "Streaming Algorithms for Robust, Real-Time Detection of DDoS Attacks," Proc. Int'l Conf. Distributed Computing Systems (ICDCS), 2007.

[16] M. Greenwald and S. Khanna, "Power-Conserving Computation of Order Statistics over Sensor Networks," Proc. Symp. Principles of Database System (PODS), 2004.

[17] V. Hristidis, L. Gravano, and Y. Papakonstantinou, "Efficient IRStyle Keyword Search over Relational Databases," Proc. Int'l Conf. Very Large Data Bases (VLDB), 2003.

[18] G. Iwerks, H. Samet, and K. Smith, "Continuous k-Nearest Neighbor Queries for Continuously Moving Points with Updates," Proc. Int'l Conf. Very Large Data Bases (VLDB), 2003.

[19] A. Jain, E. Chang, and Y. Wang, "Adaptive Stream Resource Management Using Kalman Filters," Proc. ACM SIGMOD, 2004.

[20] P. Juang, H. Oki, Y. Wang, M. Martonosi, L. Peh, and D. Rubenstein, "Energy-Efficient Computing for Wildlife Tracking: Design Tradeoffs and Early Experiences with ZebraNet," Proc. Ann. Conf. Architectural Support for Programming Languages and Operating Systems (ASPLOS-X), 2002.

[21] S. Khanna and W. Tan, "On Computing Functions with Uncertainty," Proc. Symp. Principles of Database System (PODS), 2001.

[22] N. Koudas, B. Ooi, K. Tan, and R. Zhang, "Approximate NN Queries on Streams with Guaranteed Error/Performance Bounds," Proc. Int'l Conf. Very Large Data Bases (VLDB), 2004. 
[23] Lawrance Berkeley National Laboratory, The Internet Traffic Archive, http://ita.ee.lbl.gov, 2009.

[24] O. Landsiedel, K. Wehrle, and S. Gotz, "Accurate Prediction of Power Consumption in Sensor Networks," Proc. IEEE Workshop Embedded Networked Sensors (EmNetS II), 2005.

[25] Z. Liu, K.C. Sia, and J. Cho, "Cost Efficient Processing of Min/Max Queries over Distributed Sensors with Uncertainty," Proc. ACM Symp. Applied Computing (SAC), 2005.

[26] K. Mouratidis et al., "A Threshold-Based Algorithm for Continuous Monitoring of $\mathrm{k}$ Nearest Neighbors," IEEE Trans. Knowledge and Data Eng., vol. 17, no. 11, pp. 1451-1464, Nov. 2005.

[27] J. Ni and C.V. Ravishankar, "Probabilistic Spatial Database Operations," Proc. Int'l Symp. Advances in Spatial and Temporal Databases (SSTD), 2003.

[28] C. Olston, J. Jiang, and J. Widom, "Adaptive Filters for Continuous Queries over Distributed Data Streams," Proc. ACM SIGMOD, 2003.

[29] V. Poosala and V. Ganti, "Fast Approximate Query Answering Using Precomputed Statistics," Proc. Int'l Conf. Data Eng. (ICDE), 1999.

[30] A. Silberstein, R. Braynard, and J. Yang, "Constraint Chaining: On Energy-Efficient Continuous Monitoring in Sensor Networks," Proc. ACM SIGMOD '06, 2006.

[31] N. Tatbul et al., "Load Shedding in a Data Stream Manager," Proc. Int'l Conf. Very Large Data Bases (VLDB), 2003.

[32] Y.-C. Tu, S. Liu, S. Prabhakar, and B. Yao, "Load Shedding in Stream Databases: A Control-Based Approach," Proc. Int'l Conf. Very Large Data Bases (VLDB), 2006.

[33] Mesquite Software, CSIM 19, http://www.mesquite.com, 2009

[34] S. Vrbsky and J. Liu, "Producing Approximate Answers to Setand Single-Valued Queries," J. Systems and Software, vol. 27, no. 3, pp. 243-251, 1994.

[35] O. Wolfson, P. Sistla, S. Chamberlain, and Y. Yesha, "Updating and Querying Databases That Track Mobile Units," Distributed and Parallel Databases, vol. 7, no. 3, pp. 257-387, 1999.

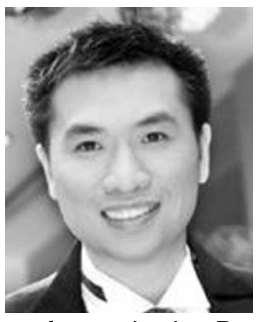

Reynold Cheng received the BEng degree in computer engineering and the MPhil in computer science and information systems from the University of Hong Kong (HKU) in 1998 and 2000, respectively, and the MSc and PhD degrees from the Department of Computer Science, Purdue University, in 2003 and 2005, respectively. $\mathrm{He}$ is an assistant professor in the Department of Computer Science at HKU. From 2005 to 2008, he was an assistant professor in the Department of Computing at Hong Kong Polytechnic University, where he received two Performance Awards. He is a member of the IEEE, the ACM, the ACM SIGMOD, and the UPE. He has served on the program committees and review panels for leading database conferences and journals like VLDB, ICDE, and TODS. He is also a guest editor for a special issue in TKDE. His research interests include database management, as well as querying and mining of uncertain data.

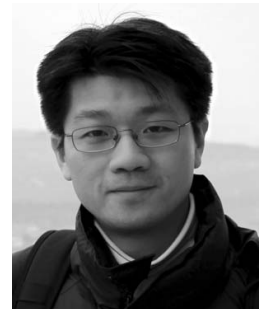

Ben C.M. Kao received the BSc degree in computer science from the University of Hong Kong in 1989, and the PhD degree in computer science from Princeton University in 1995. From 1989 to 1991, he was a teaching and research assistant at Princeton University. From 1992 to 1995, he was a research fellow at Stanford University. He is currently an associate professor in the Department of Computer Science at the University of Hong Kong. His research interests include database management systems, data mining, realtime systems, and information retrieval systems.

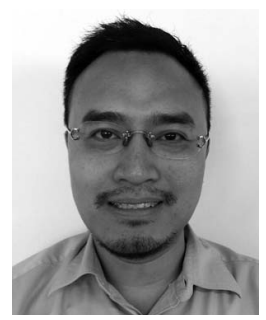

Alan Kwan received the BSc (Hons) degree in computer studies from the City University of Hong Kong in 2002, and the MPhil degree from the University of Hong Kong in 2007. He is currently a solution architect manager at an information technology company in Hong Kong. $\mathrm{He}$ is interested in the area of continuous query evaluation.

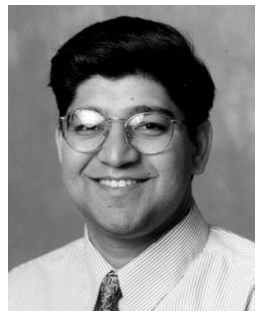

Sunil Prabhakar received the Bachelor of Technology degree in electrical engineering from the Indian Institute of Technology, Delhi, in 1990, and the $\mathrm{MS}$ and $\mathrm{PhD}$ degrees in computer science from the University of California, Santa Barbara, in 1998. He is an associate professor of computer sciences at Purdue University. His current research interests are in uncertain databases, sensor and streams databases, data privacy, and scientific databases. He is a recipient of the US National Science Foundation (NSF) CAREER Award. He serves on the editorial board for the Distributed and Parallel Databases Journal and has served on the program committees for leading database conferences such as VLDB, SIGMOD, and ICDE.

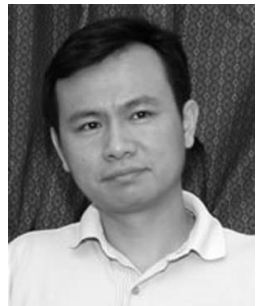

Yi-Cheng Tu received the bachelor's degree in horticulture from Beijing Agricultural University, China, and the MS and PhD degrees in computer science from Purdue University in 2003 and 2007, respectively. He is currently an assistant professor in the Department of Computer Science and Engineering at the University of South Florida. His current research efforts address energy-efficient query processing, selftuning databases, and scientific data processing. He had also worked on data stream management systems, peerto-peer systems, and multimedia databases. $\mathrm{He}$ is a member of the IEEE and the ACM.

$\triangleright$ For more information on this or any other computing topic, please visit our Digital Library at www.computer.org/publications/dlib. 